This document is the accepted manuscript version of the following article:

Tsujino, S., Helfenstein, P., Kirk, E., Voge1, T., Escher, C., \& Fink, H. W. (2010). Field-emission characteristics of molded molybdenum nanotip arrays with stacked collimation gate electrodes. IEEE Electron Device Letters, 31(9), 1059-1061. https://doi.org/10.1109/LED.2010.2052013

\title{
Field-emission characteristics of molded molybdenum nano-tip arrays with stacked collimation gate electrodes
}

\author{
S. Tsujino, Senior Member, IEEE, P. Helfenstein, E. Kirk, T. Vogel, C. Escher, and H.-W. Fink
}

\begin{abstract}
Double-gate field-emission characteristics of metallic field-emitter array (FEA) cathodes fabricated by molding with stacked collimation gate electrodes with planar end plane are reported. Collimation of the field emission electron beam with minimal reduction of the emission current was demonstrated when a negative bias was applied to the collimation gate, whereas when the two electrodes were at the same potential, the emission characteristic of the double-gate device was same as that of the single-gate device that shows emission current of $\sim 1 \mathrm{~mA}$ from 40x40 tip arrays. The results indicate that the device structure of the fabricated double-gate FEAs is promising for high-brilliance cathode applications.
\end{abstract}

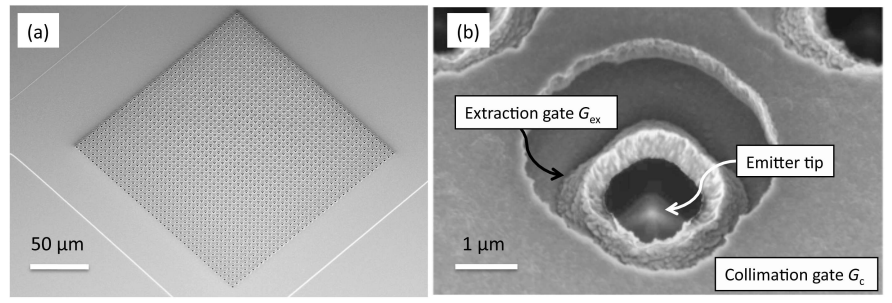

Fig. 1. (a) Scanning electron microscope image of the double-gate FEA cathode with $40 \times 40$ tips. The emitters are aligned with $5 \mu \mathrm{m}$-pitch. (b) High resolution image of one of the emitters from (a). The apex of the molybdenum emitter can be seen as the bright spot inside the extraction gate aperture.

Index Terms - Electron emission, metallic emitters, double-gate field-emitter arrays, collimation, high-brilliance, molding

\section{INTRODUCTION}

DOUBLE-gate field-emitter-array (FEA) cathodes having a collimation gate electrode $G_{\mathrm{c}}$ stacked on top of the electron extraction gate electrode $G_{\text {ex }}$ have been studied in the past for the purpose of eliminating pixel-to-pixel crosstalk in field-emitter displays [1]-[3], for field-ionizer applications [4], and for electron-beam lithography applications [5]-[7]. FEAs have also been studied as the cathode for a compact free electron laser with sub-nanometer wavelength [8]: FEAs can be competitive with the state-of-the-art photocathode [9], [10] when the angular spread $\Delta \theta$ of individual beams is reduced below $\sim 1^{\circ}$ while keeping the average current density above $\sim 1 \mathrm{kA} \mathrm{cm}^{-2}$ as demonstrated in single-gate devices [11]. $\Delta \theta$ can be reduced in double-gate FEAs by applying a negative bias $V_{c}$ to $G_{\mathrm{c}}$ with magnitude comparable to the positive electron extraction bias $V_{e x}$ applied between $G_{\text {ex }}$ and emitters as reported in the literatures [1-7]. However, since the negative $V_{c}$ can reduce the electric field $F_{a p x}$ at the tip-apex and the emission current, the optimization of the device structure minimizing the influence of $V_{c}$ on $F_{a p x}$ is crucial. For the high-brilliance applications in an acceleration gradient of the order of $100 \mathrm{MV} / \mathrm{m}$, device structures with minimal protrusion are preferred to prevent the parasitic breakdown. Our previous approach [12] based on the molded FEAs having the stacked double-gate electrodes showed successful operation of the device but the emission current decreased substantially by negative $V_{\mathrm{c}}$. In this Letter, we therefore explore the improved field-emission current-voltage characteristics of double-gate FEAs in a modified gate aperture geometry.

\section{SAMPLE AND EXPERIMENT}

We fabricated single-gate FEA devices, SG1 and SG2, and a double-gate FEA DG having $40 \times 40$ tip array. SG1 was fabricated using a FEA wafer with apex diameter $a_{\text {apx }}$ of $\sim 10 \mathrm{~nm}$. SG2 and DG were fabricated using a FEA wafer with $a_{\text {apx }}$ of $\sim 20 \mathrm{~nm}$. In addition, a $4 \times 4$ tip double-gate FEA was fabricated together with DG. The FEA wafers were fabricated by the molding method [12]-[15] supported by $0.4 \mathrm{~mm}$-thick electro-plated nickel. The emitters have $1.5 \mu \mathrm{m}$-square base size and $\sim 1.2 \mu \mathrm{m}$-height, aligned with $5 \mu \mathrm{m}$-pitch in the arrays. $G_{\text {ex }}$ layer was $0.5 \mu \mathrm{m}$-thick Mo film separated from the arrays by $1.2 \mu \mathrm{m}$-thick $\mathrm{SiO}_{2}$ film deposited by plasma-enhanced chemical vapor deposition. For the double-gate FEAs, $G_{\mathrm{c}}$ layer of $0.5 \mu \mathrm{m}$-thick Mo was added on

Manuscript received May 6, 2010. This work was supported in part by the SwissFEL project, Paul Scherrer Institute and in part by the Swiss National Science Foundation No. 200021-125084.

S. Tsujino (e-mail: soichiro.tsujino@psi.ch), P. Helfenstein, E. Kirk, and T. Vogel are with the Laboratory for Micro- and Nanotechnology, Paul Scherrer Institut, CH-5232 Villigen-PSI, Switzerland.

C. Escher and H.-W. Fink are with Physics Institut, University Zürich, Winterthurestrasse 190, CH-8057 Zürich, Switzerland. 


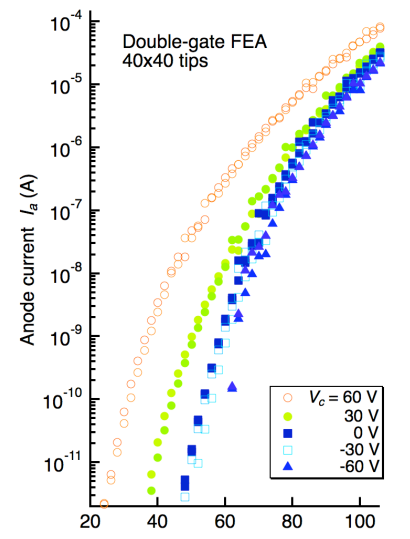

(a)

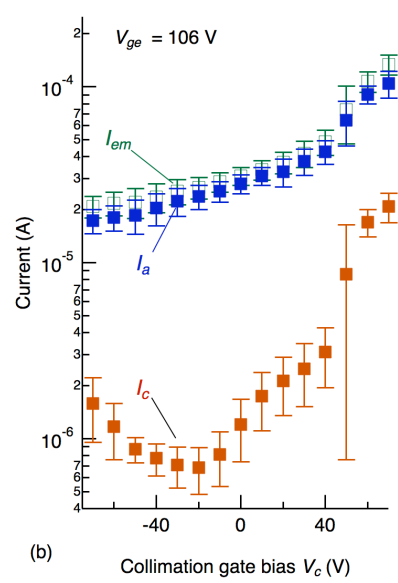

Fig. 2. Field-emission characteristics of the double-gate FEA DG with $40 \times 40$ emitter tips. (a) Anode current $I_{\mathrm{a}}$ as a function of the bias voltage $V_{\text {ge }}$ applied between the extraction gate $G_{\mathrm{ex}}$ and the emitter for several collimation gate bias $V_{\mathrm{c}}$ between 60 and $-60 \mathrm{~V}$. (b) The variation of $I_{\mathrm{a}}$, the emitter current $I_{\mathrm{em}}$, and the collimation gate current $I_{\mathrm{c}}$, when $V_{\mathrm{c}}$ was varied between -70 and $70 \mathrm{~V}$ when $V_{\text {ge }}$ was fixed at $106 \mathrm{~V}$.
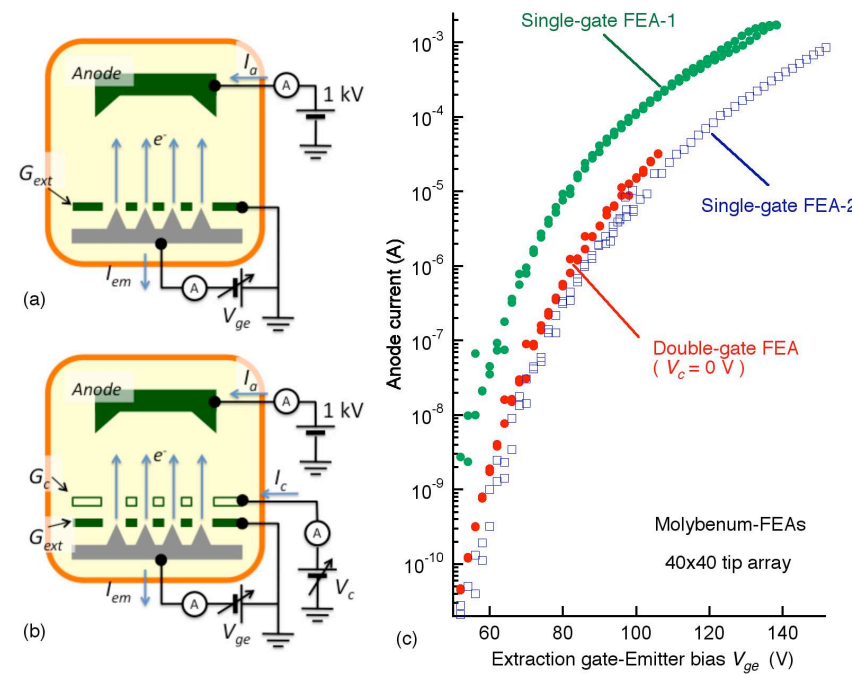

Fig. 3. Schematic diagram of the measurement setup of single-gate FEAs (a) and double-gate FEA (b). The FEAs and the anode (separated by $10 \mathrm{~mm}$ ) were mounted in the vacuum chamber (background pressure of $\sim 10^{-9}$ mbar), represented by the enclosed area. (c) Anode current $I_{\mathrm{a}}$ vs extraction gate-emitter bias $V_{\text {ge }}$ for two single-gate FEAs, SG1, SG2, and and the double-gate FEA, DG with the bias $V_{\mathrm{c}}$ at the collimation gate $G_{\mathrm{c}}$ fixed at $0 \mathrm{~V}$. All the devices have $40 \times 40$ emitters. SG1 was fabricated using an emitter array with emitter apex diameter of $\sim 10 \mathrm{~nm}$. SG2 and DG were fabricated

usino arravs with emitter anex diameter.of $\widetilde{T}^{20}$ nm
emitter array as DG and exhibited a reduction of $I_{\mathrm{a}}$ by a factor top of the extraction gate separated by $1.2 \mu \mathrm{m}$-thick SiON. The diameter of $G_{\text {ex }}$ apertures of SG1 and SG2 were equal to $2.3 \pm 0.1$ $\mu \mathrm{m}$. The aperture diameters of DG were equal to $1.2 \pm 0.1 \mu \mathrm{m}$ for $G_{\text {ex }}$ and $3.5 \pm 0.1 \mu \mathrm{m}$ for $G_{\mathrm{c}}$, respectively. The details of the fabrication procedure were described elsewhere [12].

The field-emission characteristics were measured in the setups shown in Fig. 3 (a) and (b). The field-emission microscopy experiment was conducted in a separate dedicated system, where the electron beam was amplified by the multi-channel plate and imaged by a phosphor screen, Fig. 4(a). The screen assembly was separated from the devices by $30 \mathrm{~mm}$.

\section{RESULTS AND DISCUSSIONS}

Fig. 2 (a) shows the $I_{\mathrm{a}}-V_{\mathrm{ge}}$ characteristics of $\mathrm{DG}$ for $V_{\mathrm{c}}$ between -60 and $60 \mathrm{~V}$ measured in the setup shown in Fig. 3(a) and (b). When we increased $V_{\mathrm{c}}$ negatively, the $I_{\mathrm{a}}-V_{\mathrm{ge}}$ characteristic shifts towards the larger $V_{\text {ge }}$ direction because of the decrease of $F_{\text {apx }}$ with negative $V_{c}$. However, the sensitivity of $I_{\mathrm{a}}$ to $V_{\mathrm{c}}$ is five orders of magnitude weaker than that to $V_{\text {ge. }}$. Fig. 2 (b) shows $I_{\mathrm{a}}$, the current $I_{\mathrm{em}}$ injected to the emitter substrate and the current $I_{\mathrm{c}}$ through $G_{\mathrm{c}}$. We observe tendencies that $I_{\mathrm{c}}$ increases faster than $I_{\mathrm{a}}$ and $I_{\mathrm{em}}$ for positive $V_{\mathrm{c}}$ and a slight increase of Ic with the decrease of $V_{\mathrm{c}}$ for $V_{\mathrm{c}}$ below $-20 \mathrm{~V}$. The former can be ascribed to the increased capture of the field emission electrons by $G_{\mathrm{c}}$ while the latter can be ascribed a field emission from the $G_{\text {ex }}$ edges to $G_{\mathrm{c}}$ as observed in Ref. 16]. Neverthless, $I_{\mathrm{c}}$ as well as the difference between $I_{\mathrm{em}}$ and $I_{\mathrm{a}}$ are less than $5 \%$ of $I_{\mathrm{a}}$ for $V_{\mathrm{c}}$ below $0 \mathrm{~V}$; the capture of the field-emission electrons by $G_{\text {ex }}$ and $G_{\mathrm{c}}$ is minimal and that the gate leak currents are small.

The observed emission current characteristic fits well to the equation, $I_{\mathrm{a}}=A V^{\mathrm{n}} \exp (-B / V)$, with $n$ equal to $2[17]$ and with the total effective bias voltage $V$ equal to $\left(V_{\mathrm{ge}}+\gamma V_{\mathrm{c}}\right)$, where $\gamma$ is the contribution of $V_{\mathrm{c}}$ to the apex field. From the result of Fig. 2, we evaluated $\gamma$ to be equal to $(0.17 \pm 0.014)$. The evaluation error represents the bound that the rms spread of the quantity $\ln \left(I_{\mathrm{a}} / V^{2}\right)$ is below $4 \%$ when $V$ is equal to $60 \mathrm{~V}$ for $V_{\mathrm{c}}$ between -70 and +70 $\mathrm{V}$. The observed value of $\gamma$ is comparable to the theoretical parameter $\gamma^{\text {(th) }}$ given by $\left(1+D_{\mathrm{c}} / D_{\mathrm{ex}}\right)^{-1}$ equal to $0.18 \pm 0.01$ obtained from the device geometry, where $D_{\mathrm{ex}}$ and $D_{\mathrm{c}}$ are the distances between the emitter apex and $G_{\mathrm{ex}}$ and $G_{\mathrm{c}}$, respectively. Here, $\gamma^{\text {(th) }}$ was derived by assuming that $F_{\text {apx }}$ is proportional to $\left[V_{\mathrm{ge}} / D_{\mathrm{ex}}+\right.$ $\left.\left(V_{\mathrm{c}}+V_{\mathrm{ge}}\right) / D_{\mathrm{c}}\right]$. We also note that the previously reported double-gate device [12], that was fabricated from the same $\gamma$-value than the present device. This is consistent with the above analysis.

In Fig. 3 (c) we compare the $I_{\mathrm{a}}-V_{\text {ge }}$ characteristics of DG with $V_{\mathrm{c}}$ equal to $0 \mathrm{~V}$ with two single-gate devices. All three devices have $40 \times 40$ emitter arrays. We observed that $I_{\mathrm{a}}$ of the single-gate devices reach $\sim 1 \mathrm{~mA}$ at $V_{\text {ge }}$ of 130-150 V. The maximum $I_{\mathrm{a}}$ of DG was somewhat lower due to the premature failure of the device but its $I_{\mathrm{a}}-V_{\text {ge }}$ characteristic is same as that of SG2 within $\sim 5 \mathrm{~V}$ of $V_{\text {ge. }}$. This shows the uniformity of the single- and double-gate fabrication processes over the $40 \times 40$ tips.

Finally, to study the effect of $V_{\mathrm{c}}$ on the electron beam collimation, we measured the beam profile in low current regime, Fig. 4, using the double-gate device having $4 \times 4$ emitters. Similarly to the large array emitter, the decrease of $I_{\mathrm{em}}$ for the $4 \times 4$ emitter array was $20 \%$ when $V_{\mathrm{c}}$ was decreased from 0 to $-70 \mathrm{~V}$, Fig.4 (d). Fig. 4 (b) shows that when $V_{\text {ge }}$ was fixed at $86 \mathrm{~V}$, the beam exhibited the emission angle $\Delta \theta$ of $(20 \pm 3)^{\circ}$ for $V_{\mathrm{c}}$ larger than $-30 \mathrm{~V} . \Delta \theta$ was evaluated from the full-width at the half maximum size of the intensity distribution of the phosphor screen image and the screen-FEA distance $D$. This value is consistent with the previous observation for single-gate Spindt-type FEAs [18], [19]. When $V_{\mathrm{c}}$ was further decreased to $-62 \mathrm{~V}, \Delta \theta$ was decreased to $(2.3 \pm 0.4)^{\circ}$ in one direction. The asymmetry and distortion of the collimated beam shape should be improved by careful design of the electrode shape [20] and by elimination of the parasitic field due to the screen assembly, the extraction gate, the electrical 
contact assembly of the FEA mount, and the aperture shapes in the future experiment. Detailed analysis of the observed collimation characteristic and its comparison with theory will be described elsewhere [21].

In summary, we showed that by engineering the aperture sizes it is possible to collimate the field emission electron beam while minimizing the emission current reduction in double-gate FEAs with stacked $G_{\mathrm{c}}$ with planar end plane. Further optimization of the device structures such as the gate electrode thicknesses [7], the gate insulator thicknesses, and the gate aperture sizes are the next subjects of the research.

\section{ACKNOWLEDGMENT}

The authors acknowledge A. F. Wrulich, J. Gobrecht, and H.-H. Braun for their support and helpful discussions on the FEA applications for FEL, B. Haas, J. Lehmann, and A. Weber for their technical help for the FEA fabrications, and M. Dehler
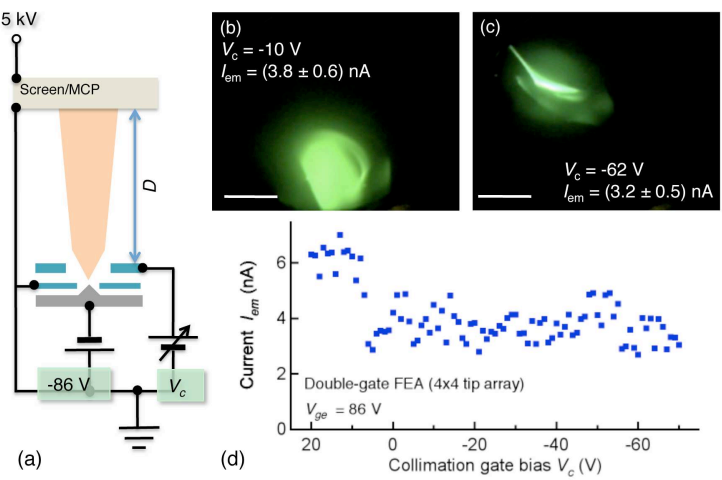

Fig. 4. (a) Schematic diagram of the electron beam imaging experiment setup for the double-gate FEA with $4 \times 4$ emitters with fixed $V_{\text {ge }}$ of $86 \mathrm{~V}$. (b) and (c) show the result for $V_{\mathrm{c}}$ equal to $-10 \mathrm{~V}$ and $-62 \mathrm{~V}$, respectively, when the distance $D$ between FEA and screen was equal to $30 \mathrm{~mm}$. The bars indicate 10 $\mathrm{mm}$ length on the screen. (d) The relation between $I_{\mathrm{em}}$ and $V_{\mathrm{c}}$ during the measurement.

and S. C. Leemann for the discussions on the emittance modeling.

\section{REFERENCES}

[1] W. D. Kesling, and C. E. Hunt, "Beam focusing for field-emission flat-panel displays", IEEE Trans. ED, vol. 42, Feb. 1995, pp. 340-347.

[2] J. Itoh, Y. Tohma, K. Morikawa, S. Kanemaru, and K. Shimizu, "Fabrication of double-gated Si field emitter arrays for focused electron beam generation", J. Vac. Sci. Technol. B, vol. 13, 1995, pp. 1968-1972.

[3] L. Dvorson, M. Ding, and A. I. Akinwande, “Analytical electrostatic model of silicon conical field emitters - Part I", IEEE Trans. ED, vol. 48, Jan. 2001, pp. 134-143.

[4] L.-Y. Chen, L. F. Velasquez-Garcia, X. Wnag, K. Teo, and A. I. Akinwande, "A micro ionizer for portable mass spectrometers using double-gated isolated vertically aligned carbon nanofiber arrays", IEDM 2007 Technical digest, 2007, pp. 843-846.

[5] L. R. Baylor et al. "Digital electrostatic electron-beam array lithography" J. Vac. Sci. Technol. B , vol. 20, 2002, pp. 2646-2650.

[6] Y. Neo et al. "Focusing characteristics of double-gated field-emitter arrays with a lower height of the focusing electrode" Appl. Phys. Express, vol. 1, 2008, pp.053001.

[7] A. Hosono, S. Kawabuchi, S. Horibata, S. Okuda, H. Harada, and M. Takai, "High emission current double-gated field emitter arrays" J. Vac. Sci. Technol. B vol. 17 (1999) pp. 575-579.

[8] B. D. Patterson et al. "Coherent science at the SwissFEL X-ray laser" to be published in New J. Phys. (2010).

[9] M. Dehler, "Design and modeling of field emitter arrays for a high brilliance electron source" Proc. the $9^{\text {th }}$ International Computational Accelerator Physics Conference (ICAP2006), Oct. 2-6, 2006, Chamonix, France, TUPP10, pp. 114-117.

[10] Y. Ding et al. "Measurements and simulations of ultralow emittance and ultrashort electron beams in the linac coherent light source" Phys. Rev. Lett. vol. 102,2009 , pp. 254801.

[11] P. R. Schwoebel, C. A. Spindt, and C. E. Holland, "High current, high current density field emitter array cathodes", J. Vac. Sci. Technol. B 23, 2005, pp. 691-693.

[12] E. Kirk, S. Tsujino, T. Vogel, J. Gobrecht, and A. Wrulich, "Fabrication of all-metal field emitter arrays with controlled apex sizes by molding" J. Vac. Sci. Techol. B, vol. 27, 2008, pp. 1813-1820.

[13] H. F. Gray, R. F. Greene, "Method of manufacturing a field-emission cathode structure", U. S. Pat. No. 4,307,507 issued Dec. $29,1981$.

[14] M. Nakamoto, T. Hasegawa, T. Ono, T. Sakai, N. Sakuma, "Low operation voltage field emitter arrays using low work function materials fabricated by transfer mold technique" Technical Digest, International Electron Devices Meeting, 1996, Dec 1996, 12-3-1, pp. 297 - 300.

[15] K. Subramanian et al. "Enhanced electron field emission from micropatterned pyramidal diamond tips incorporating CH4/H2/N2 plasma-deposited nanodiamond" Diamond Relat. Mater. 15, 2006, pp. 1126-1131.

[16] M. Nagao, T. Yoshida, S. Kanemaru, Y. Neo, and H. Mimura, "Fabrication of a field emitter array with a built-in einzel lens" Jpn. J. Appl. Phys. vol. 48, 2009, pp. 06FFK02.

[17] C. A. Spindt, I. Brodie, L. Humphrey, "Physical properties of thin-film field emission cathodes with molybdenum cones" J. Appl. Phys. Vol. 47 (1976) pp.5248-5263.

[18] P. M. Phillips, C. Hor, L. Malsawma, K. L. Jensen, and E. G. Zaidman, "Design and construction of apparatus for characterization of gated field emitter array electron emission" Rev. Sci. Instrum. vol. 67, June 1996, pp. 2387- 2393.

[19] S. C. Leemann, A. Straudel, and A. Wrulich, "Beam characterization for the field-emitter-array cathode-based-low-emittance gun" Phys. Rev. ST. Accel. Beams 10, 2007, pp. 071302.

[20] C. Py, J. Itoh, T. Hirano, and S. Kanemaru, "Beam focusing characteristics of silicon microtips with an in-plane lens" IEEE Trans. ED, vol. 44, March 1997, pp. 498-502.

[21] P. Helfenstein et al. unpublished (2010). 